\title{
The clinical value of platelet parameters combined with sFlt-1/ PIGF in predicting preeclampsia
}

\author{
Yan $\mathrm{Li}^{1}$, Lifang Sun ${ }^{1}$, Xiuli Zheng ${ }^{1}$, Jingfang Liu ${ }^{1}$, Rong Zheng ${ }^{1}$, Yanwei $\mathrm{Lv}^{2}$ \\ ${ }^{1}$ Department of Obstetrics and Gynecology, Beijing Jishuitan Hospital, Beijing, China; ${ }^{2}$ Clinical Epidemiology Research Office, Beijing Jishuitan \\ Hospital, Beijing, China \\ Contributions: (I) Conception and design: Y Li, L Sun; (II) Administrative support: L Sun, Y Lv; (III) Provision of study materials or patients: Y Li, \\ L Sun; (IV) Collection and assembly of data: Y Li, L Sun, X Zheng, Y Lv; (V) Data analysis and interpretation: Y Li, J Liu, R Zheng, Y Lv; (VI) \\ Manuscript writing: All authors; (VII) Final approval of manuscript: All authors. \\ Correspondence to: Lifang Sun, PhD. Department of Obstetrics and Gynecology, Beijing Jishuitan Hospital, No.68 Huinan North Road Huilongguan \\ Town, Changping District, Beijing 100096, China. Email: lifangsun2000@163.com.
}

Background: The aim of this study is to evaluate the association between platelet parameters and soluble
vascular endothelial growth factor receptor-1 (sFlt-1)/placenta growth factor (PlGF) in preeclampsia (PE)
and establish a prediction model by analyzing commonly used biochemical markers.
Methods: A nested case-control study involving 270 pregnant women in their second trimester from the
Beijing Jishuitan Hospital was conducted. They were divided into PE group and control group. The levels
of PlGF, sFlt-1, sFlt-1/PlGF, and platelet parameters were recorded and compared at 20-24 gestational
weeks. The correlation between platelet parameters and PlGF, sFlt-1, and sFlt-1/PLGF was then analyzed.
A receiver operating characteristic (ROC) curve was used to calculate the sensitivity, specificity, positive
predictive value (PPV), and negative predictive value (NPV) of various biomarkers in predicting PE.

Results: In PE group, the levels of mean platelet volume (MPV), platelet distribution width (PDW), sFlt-1, and sFlt-1/PLGF were higher than in the control group, while the levels of platelet count (PC), PC/MPV, and PLGF in PE group were lower. Spearman correlation analysis showed that PC and PC/ MPV were negatively correlated with sFlt-1 and sFlt-1/PLGF, and positively correlated with PLGF, while further analysis found that PC/MPV had the largest area under the ROC curve with sensitivity of $83.7 \%$ and specificity of $86.2 \%$. The area under curve (AUC) of sFlt-1, PLGF, and sFlt-1/PLGF for predicting PE were $0.731,0.772$, and 0.825 , respectively. Their AUCs could be improved to $0.820,0.838$, and 0.873 when combined with PC/MPV.

Conclusions: The accuracy of sFlt-1/PlGF in predicting the risk of $\mathrm{PE}$ in the second trimester is significantly improved when combined with PC/MPV, which is expected to be an ideal tool for PE prediction.

Keywords: Preeclampsia; platelet parameters; soluble vascular endothelial growth factor receptor-1; platelet count; placenta growth factor

Submitted Apr 20, 2021. Accepted for publication Jun 16, 2021.

doi: 10.21037/apm-21-1244

View this article at: https://dx.doi.org/10.21037/apm-21-1244

\section{Introduction}

Preeclampsia (PE) is an idiopathic disease of pregnancy, which is mainly manifested as hypertension and proteinuria after 20 weeks of pregnancy. It is still one of the main causes of maternal and infant mortality (1-4). When clinical symptoms appear, pregnant women and fetuses have suffered varying degrees of damage. Particularly, patients with early-onset severe PE have an early onset and a poor prognosis. Therefore, early prediction of high-risk patients 
with $\mathrm{PE}$ has important clinical significance for disease treatment and improvement of pregnancy outcomes $(5,6)$. Several factors have been thought to contribute to PE, involving immune, genetic, endothelial cell dysfunction, and coagulation dysfunction, which eventually results in increased resistance of uterine arterial blood flow, inadequate placental perfusion, and decreased function $(7,8)$. Recently, studies have found that serum markers such as soluble vascular endothelial growth factor receptor-1 (sFlt-1), placenta growth factor (PlGF), soluble endothelial factor, serum placental protein 13 , and vascular endothelial growth factor have the potential to predict PE. Among them, the sFlt-1 and PlGF have been extensively studied (9). Additionally, some studies found the sFlt-1/PIGF ratio could better reflect the antiangiogenic activity and it is also a better predictor of $\mathrm{PE}$ than either measure alone $(10,11)$. However, further research is needed to improve the accuracy of PE prediction. With the rapid development of PE etiology research, more etiology-related indicators have been found, but there is no prediction model with high sensitivity and specificity that can be used in the clinic, and the differences between the indexes in different research are obvious (12). Recent studies suggested that platelet aggregation plays a crucial role in the development of the disease. The contact of platelets with injured endothelium activates the coagulation system, resulting in increased platelet consumption and bone marrow production. As a result, bone marrow releases platelets causing an increase in platelet parameters. While studies have confirmed that platelet parameters such as platelet count (PC), mean platelet volume (MPV), platelet distribution width (PDW), and PC to MPV ratio, can be utilized to predict PE (13-15), the relationship between these and $\mathrm{PE}$ requires more research. There are few studies on the predictive efficacy of platelet parameters, sFlt-1 and PlGF in PE. The purpose of this study was to evaluate the association between platelet parameters and sFlt-1/PlGF in PE and establish an appropriate prediction method by analyzing commonly used biochemical markers as a means of reducing future risks of PE-related death and disease. We present the following article in accordance with the STARD reporting checklist (available at https://dx.doi.org/10.21037/apm-21-1244).

\section{Methods}

All procedures performed in this study involving human participants were in accordance with the Declaration of Helsinki (as revised in 2013). The study was approved by the Ethics committee of Beijing Jishuitan Hospital and informed consent was taken from all the patients.

\section{Patients}

A total of 2,573 pregnant women with singleton pregnancy and in their second trimester attended the Obstetrics and Gynecology department of Beijing Jishuitan Hospital from April 2018 to April 2020. The age range was 24 to 40 years, and the gestational age at sampling was 30 to 42 weeks. We excluded patients with diseases that affect placental perfusion and platelet parameters. According to the pregnancy outcome, all patients were divided into PE group and control group for a nested case-control study. The control group was defined as age-matched single pregnant women without hypertension, diabetes, hyperthyroidism, and other pre-pregnancy complications.

\section{Biomarker detection}

Baseline data were collected during hospitalization, including age, systolic blood pressure (SBP), diastolic blood pressure (DBP), body mass index (BMI), gestational age at sampling, nulliparity and vaginal delivery proportion. At 20-24 weeks gestation, $4 \mathrm{~mL}$ blood samples were obtained and platelet parameters were measured by automated hematology analyzer. The concentrations of PLGF and sFlt-1 were measured by an ELISA kit purchased from Shanghai enzyme-linked Biotechnology Co., Ltd.

\section{Clinical definitions}

PE was defined as a normotensive woman with blood pressure $\geq 140 / 90 \mathrm{mmHg}$ and proteinuria after 20 weeks of pregnancy. Severe PE was defined if any of the following was met: (I) systolic blood pressure $\geq 160 \mathrm{mmHg}$ or diastolic blood pressure $\geq 110 \mathrm{mmHg}$; (II) platelet count $<100,000 / \mu \mathrm{L}$; (III) elevated liver enzymes; (IV) severe persistent right upper quadrant or epigastric pain; (V) renal insufficiency; (VI) pulmonary edema. The 10th percentile of estimated weight less than gestational age is defined as fetal growth restriction (FGR).

\section{Statistical analysis}

Student's $t$-test and Mann-Whitney U were utilized for normally distributed and skewed numerical data, respectively. Chi-square test was performed for the 
Table 1 Clinical characteristics of studied population

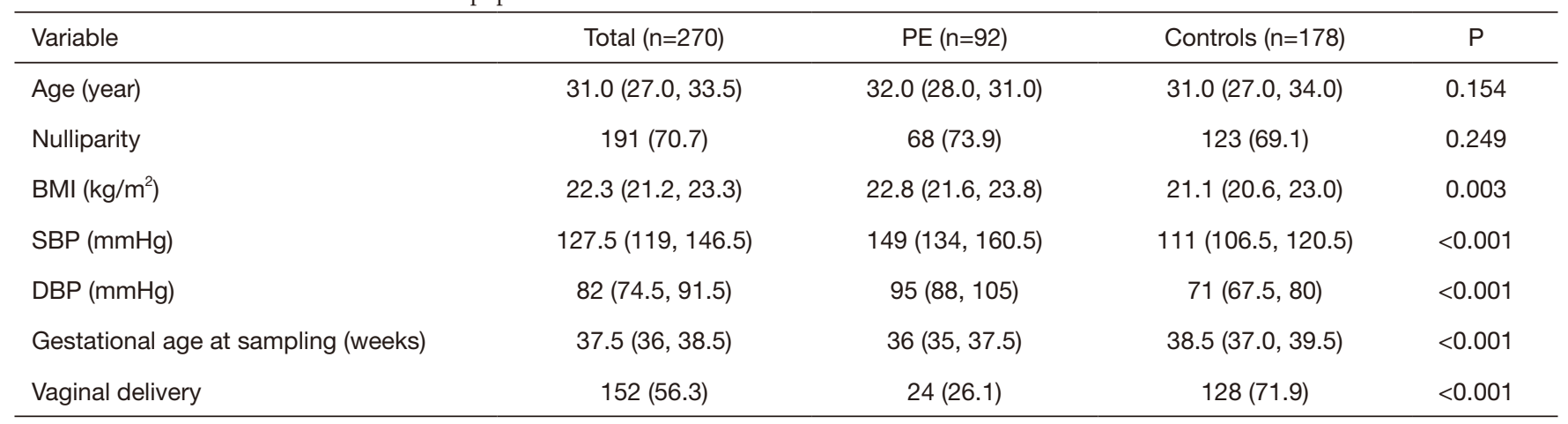

Data is expressed as medians (quartiles) or as frequency (\%). PE, preeclampsia; BMI, body mass index; SBP, systolic blood pressure; DBP, diastolic blood pressure.

comparison of categorical variables. Spearman correlation analysis was used to calculate the correlation between platelet parameters and PlGF, sFlt-1, and sFlt-1/PlGF. Receiver operating characteristic (ROC) curve was performed to analyze the sensitivity, specificity, positive predictive value (PPV), and negative predictive value (NPV) of various parameters in predicting PE. IBM SPSS Statistics 19.0, R package version 3.6.2. and Medcalc (Version 22.0.1) were used for statistical analyses.

\section{Results}

\section{Comparison of clinical characteristics between the two groups}

A total of 270 women enrolled and were divided into PE group and control group. Of the patients with PE, $41.3 \%$ were classified as severe, and the rest were mild. There was no statistical difference between the PE group and controls in age and nulliparity proportion $(\mathrm{P}>0.05)$. However, there were significant differences in BMI, SBP, DBP, gestational age at sampling and vaginal delivery between the two groups $(\mathrm{P}<0.05)$. The $\mathrm{PE}$ group had higher $\mathrm{BMI}$, SBP, DBP and markedly decreased gestational age at sampling and vaginal delivery proportion. Baseline characteristics of the two groups are shown in Table 1.

\section{Comparison of various parameters by severity of $P E$ among pregnant women}

The results showed that the levels of MPV, PDW, sFlt-1, and sFlt-1/PLGF in the PE group were significantly higher than that in the controls, while the PC, PC/MPV, and PLGF levels were significantly lower $(\mathrm{P}<0.05)$. Further analysis found that severe $\mathrm{PE}$ women had lower PC, PC/MPV, PLGF, and higher sFlt-1 and sFlt-1/PLGF, as opposed to mild $\mathrm{PE}$ and controls $(\mathrm{P}<0.05)$. However, MPV and PDW were not significantly different when mild and severe PE patients were compared. The various parameters of pregnant women with and without $\mathrm{PE}$ are shown in Table 2.

\section{Association between platelet parameters and PlGF, sFlt-1, and sFlt-1/PLGF in PE women}

$\mathrm{PE}$ was negatively correlated with sFlt-1 and sFlt-1/PlGF, while positively correlated with PlGF. Similarly, PC/MPV showed negative correlation with sFlt-1 and sFlt-1/PlGF, and positive correlation with PlGF. In pregnant women with PE, MPV, and PDW did not correlate significantly with PlGF, sFlt-1, orsFlt-1/PlGF, as shown in Table 3.

\section{Predictive value of platelet parameters for PE}

ROC curves were used to derive cutoffs for platelet parameters, as shown in Figure 1 and Table 4, and the analysis showed that the best cut-off values for PC, MPV, $\mathrm{PDW}$, and PC/MPV were $217 \times 10^{3} / \mu \mathrm{L}, 10.5 \mathrm{fL}, 11.8 \%$, and 24.2 , respectively. The area under curve (AUC) was 0.743 (95\% CI, 0.686-0.801) for PC, 0.836 (95\% CI, 0.768-0.906) for PC/MPV, 0.663 (95\% CI, 0.569-0.754) for MPV, and 0.627 (95\% CI, 0.558-0.687) for PDW. PC/MPV had the largest area under the ROC curve with sensitivity of $83.7 \%$ and specificity of $86.2 \%$, and a statistical significance was observed. 
Table 2 Comparison of different biochemical parameters in severe PE, mild PE, and control cases

\begin{tabular}{|c|c|c|c|}
\hline Variable & \multicolumn{2}{|c|}{$P E(n=92)$} & Controls $(n=178)$ \\
\hline Platelet count, $\times 10^{3} / \mu \mathrm{L}$ & $229.0(163.2-270.8)^{\star}$ & $197.2(144.7-245.9)^{\star^{\#}}$ & $263.5(220.4-335.1)$ \\
\hline Mean platelet volume, $\mathrm{fL}$ & $10.7(9.6-11.3)^{\star}$ & $11.8(9.9-13.5)^{\star}$ & $9.2(8.0-10.7)$ \\
\hline Platelet distribution width, \% & $12.3(11.6-14.2)^{*}$ & $13.1(12.6-15.0)^{*}$ & $10.6(9.8-13.5)$ \\
\hline $\mathrm{sFlt}-1, \mathrm{pg} / \mathrm{mL}$ & $1,411.0(973.6-2,056.4)^{\star}$ & $1,793 \cdot 2(1,301.7-2,414.6)^{\star^{\#}}$ & $1,353.1(843.6-1,991.4)$ \\
\hline PIGF, pg/mL & $368.2(295.2-475.4)^{\star}$ & $324.3(261.3-418.2)^{\star^{\#}}$ & $537.6(369.5-827.8)$ \\
\hline sFIt-1/PIGF & $3.7(2.6-5.8)^{\star}$ & $4.6(3.2-7.3)^{\star^{\#}}$ & $1.9(1.3-3.4)$ \\
\hline
\end{tabular}

Data is presented as medians and quartiles. Compared with the controls, ${ }^{*} \mathrm{P}<0.05$; compared with the mild, " $\mathrm{P}<0.05$. $\mathrm{PE}$, preeclampsia; sFIt-1, soluble vascular endothelial growth factor receptor-1; PIGF, placenta growth factor.

Table 3 Association between platelet parameters and PlGF/sFlt-1 at 20-24 weeks gestation

\begin{tabular}{lccc}
\hline Variable & $\begin{array}{c}\mathrm{sFlt}-1, \\
\mathrm{pg} / \mathrm{mL}\end{array}$ & $\begin{array}{c}\mathrm{PIGF}, \\
\mathrm{pg} / \mathrm{mL}\end{array}$ & $\begin{array}{c}\mathrm{sFlt}-1 / \\
\mathrm{PIGF}\end{array}$ \\
\hline Platelet count, $\times 10^{3} / \mu \mathrm{L}$ & & & \\
$\mathrm{r}$ & -0.321 & 0.415 & -0.387 \\
$\mathrm{P}$ & 0.016 & $<0.001$ & $<0.001$ \\
Mean platelet volume, fL & & & \\
r & 0.314 & -0.220 & 0.190 \\
P & 0.025 & 0.063 & 0.083 \\
Platelet distribution width, \% & & & \\
r & 0.160 & -0.086 & 0.114 \\
P & 0.092 & 0.175 & 0.128 \\
Platelet count/mean platelet & & & \\
volume & & & \\
r & -0.416 & 0.359 & -0.370 \\
P & $<0.001$ & $<0.001$ & $<0.001$ \\
\hline
\end{tabular}

\section{Predictive value of sFlt-1, PlGF, sFlt-1/PlGF, and PC/ $M P V$ for $P E$}

The area under curve (AUC) of sFlt-1/PIGF was 0.825 (95\% CI, 0.744-0.879), which was higher than that of sFlt-1 [0.731 (95\% CI, 0.692-0.840)] and PlGF [0.772 (95\% CI, 0.648-0.804)], respectively (Figure 2A). After being combined with PC/MPV, the AUC of sFlt-1, PlGF, and sFlt-1/PlGF were 0.820 (95\% CI, 0.746-0.882), 0.838 (95\% CI, 0.765-0.896), and 0.873 (95\% CI, 0.805-0.924),

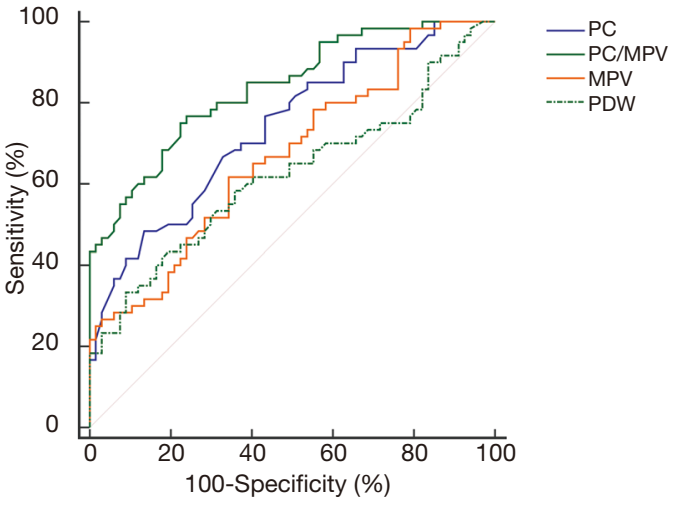

Figure 1 ROC curve of platelet parameters in pregnant women at 20-24 weeks of gestation. ROC, receiver operating characteristic.

respectively (Figure $2 B$ ). The AUC of sFlt-1/PlGF + $\mathrm{PC} / \mathrm{MPV}$ was 0.87 higher than that of sFlt-1/PlGF and PC/MPV. The results were summarized in Table 5 .

\section{Discussion}

$\mathrm{PE}$ is a common systemic disease during pregnancy, with a global incidence of $3 \%$ to $5 \%$. The clinical manifestations of PE include hypertension, proteinuria, and edema during pregnancy, and may be accompanied by multiple organ damage. It has been reported that $10-15 \%$ of maternal deaths are directly related to PE (16). There is limited evidence of biochemical indexes that predict the occurrence and prognosis of PE. One of the antiangiogenic factors involved in $\mathrm{PE}$ is sFlt-1 and the main proangiogenic factor is PlGF. The imbalance in these markers has a regulatory effect on the function of vascular endothelial cells and 
Table 4 Results of ROC analysis of platelet parameters in predicting PE

\begin{tabular}{|c|c|c|c|c|c|c|c|}
\hline Variable & Area & $95 \% \mathrm{Cl}$ & Cut-off & Sensitivity (\%) & Specificity (\%) & PPV (\%) & NPV (\%) \\
\hline Platelet count, $\times 10^{3} / \mu \mathrm{L}$ & 0.743 & $0.686-0.801$ & 217 & 73.9 & 80.6 & 66.3 & 85.6 \\
\hline Mean platelet volume, $\mathrm{fL}$ & 0.826 & $0.768-0.906$ & 10.5 & 83.7 & 86.2 & 75.8 & 91.1 \\
\hline Platelet distribution width, \% & 0.663 & $0.569-0.754$ & 11.8 & 71.5 & 61.4 & 48.9 & 80.6 \\
\hline Platelet count/mean platelet volume & 0.627 & $0.558-0.687$ & 24.2 & 78.3 & 52 & 45.8 & 82.2 \\
\hline
\end{tabular}

ROC, receiver operating characteristic; PE, preeclampsia, PPV, positive predictive value; NPV, negative predictive value
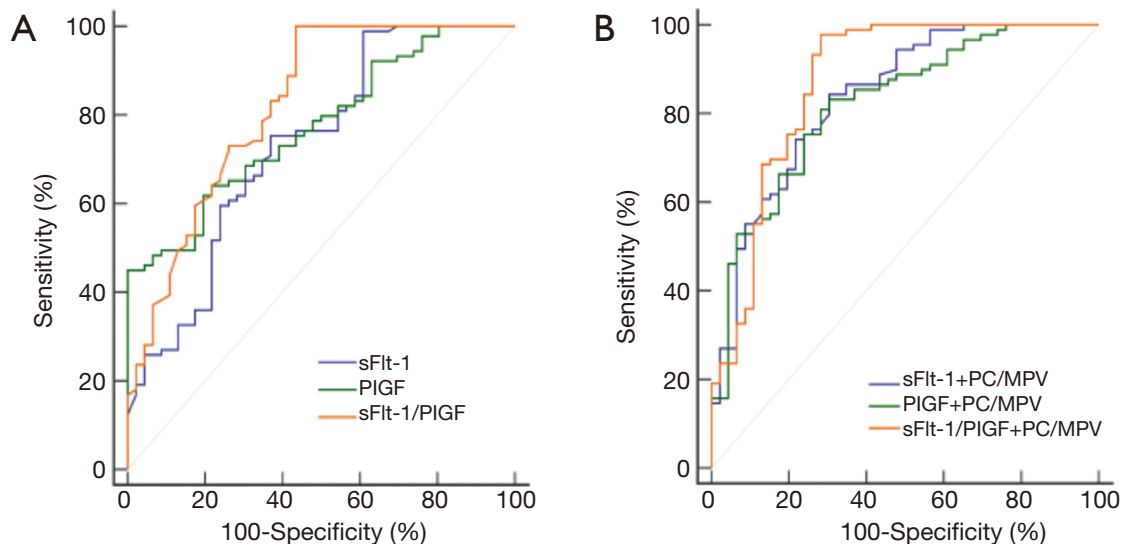

Figure 2 ROC curve analysis of predictive value of indicators for PE. (A) ROC curve of sFlt-1, PlGF and sFlt-1/PlGF; (B) ROC curve of sFlt-1, PlGF and sFlt-1/PlGF combined with PC/MPV. ROC, receiver operating characteristic; sFlt-1, soluble vascular endothelial growth factor receptor-1; PlGF, placenta growth factor; PC, platelet count; MPV, mean platelet volume; PE, preeclampsia.

Table 5 Results of ROC analysis of sFlt-1, PlGF, sFlt-1/PlGF, and PC/MPV in predicting PE

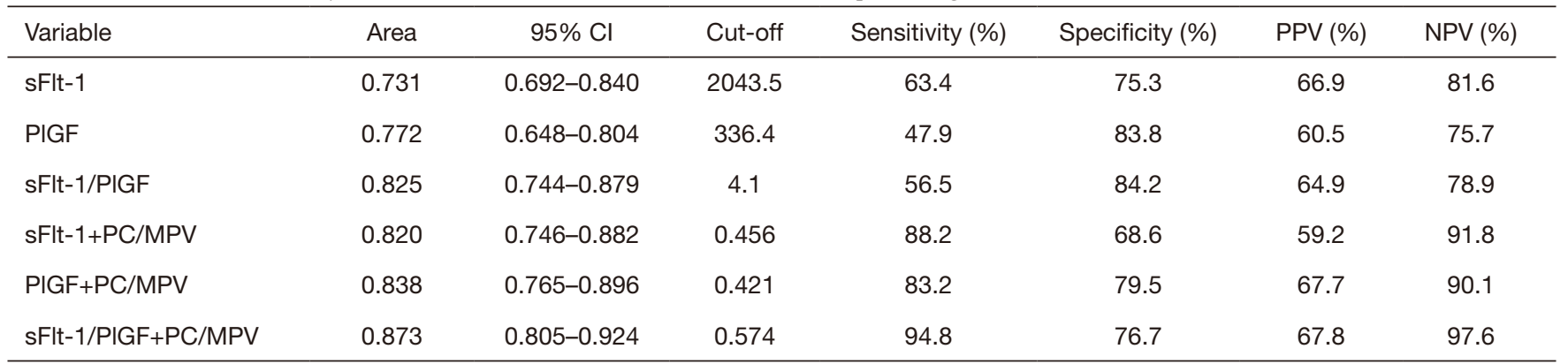

ROC, receiver operating characteristic; sFIt-1, soluble vascular endothelial growth factor receptor-1; PIGF, placenta growth factor; PC, platelet count; MPV, mean platelet volume; PE, preeclampsia; PPV, positive predictive value; NPV, negative predictive value.

may play an important role in the pathogenesis of the PE. In women with $\mathrm{PE}$, maternal serum levels of sFlt-1 are increased, and PlGF levels are significantly decreased. The degree of increase or decrease is related to the severity of $\mathrm{PE}$ and their ratio is considered to be a useful index for predicting PE.

There is intense interest in the search for biochemical indexes that are related to hematological parameters, including the neutrophil-lymphocyte ratio, red cell distribution width, and platelet parameters, which are simple procedures and easily obtainable $(17,18)$. Platelet parameters have been found to exhibit change before the onset of PE, and several researchers have implied that they can be used in the second trimester as useful biochemical 
markers for the prediction of PE $(19,20)$. However, there is a lack of comparative studies on platelet parameters and several other biomarkers of $\mathrm{PE}$. This study investigated the alterations of various biochemical indexes in women with PE and healthy pregnant women and analyzed the predictive value of the above indicators.

Common risk factors of $\mathrm{PE}$ include primipara, age $\geq 35$ years, body mass index $\geq 24 \mathrm{~kg} / \mathrm{m}^{2}$, history of hypertension and PE, and family history of PE. Quan et al. (21) noted that the risk of developing $\mathrm{PE}$ in pregnant women aged $\geq 35$ years was $2-4$ times higher than that in younger pregnant women, which may be induced by arteriosclerosis and blood pressure elevation in older patients. Extensive research confirmed that chronic hypertension is an independent risk factor for PE, especially in cases with systolic blood pressure $>130 \mathrm{mmHg}$ at the first prenatal check-up (22). An increase in blood pressure usually produces pathologic changes to the placenta, small blood vessel spasm, and an increase in peripheral resistance, which then causes endothelial cell injury and PE. In this study, the proportion of $\mathrm{PE}$ patients with systolic blood pressure $>130 \mathrm{mmHg}$ was significantly higher than that of the controls, and the diastolic blood pressure of the controls was significantly lower than that of the PE group. Risk factors such as hypertension, overweight, and advanced age can indicate the occurrence of $\mathrm{PE}$ to a certain extent but have poor capability in predicting PE. Due to the rapid disease progression, more stable and precise indexes are required for clinical prediction.

The detection of biochemical parameters is an important method for predicting $\mathrm{PE}$ as it can determine a credible early disease assessment during the asymptomatic stage and effectively monitor high-risk groups. Changes in platelet parameters are one of the most commonly identified hematological changes in PE. In our study, PC and PC/ MPV showed a significant decrease, while MPV and PDW increased significantly in $\mathrm{PE}$ women compared to controls, suggesting that the above parameters are valuable in predicting the occurrence of $\mathrm{PE}$.

The role of sFlt-1 and PLGF in the identification of women with preeclampsia has been largely reported, and during pregnancy, the placenta secretes large amounts of PlGF and sFlt-1. As a decoy receptor for VEGF and PlGF, increased sFlt-1 binds to free VEGF and PlGF in maternal circulation and inhibits the biological actions of their membrane receptor (23-25). Studies by Cui et al. (26) showed that PlGF and sFlt-1 are important biomarkers for predicting the occurrence of preeclampsia and its complications. In addition, the result also showed that the sFlt-1/PlGF ratio is a useful triage tool for the pregnancy outcome of patients with $\mathrm{PE}$, and its predictive performance is better than the individual marker. In this study, compared with pregnant women in the control group, patients who developed PE had significantly higher serum sFlt-1 concentration, lower PlGF concentration, and higher PlGF/ sFlt-1 ratio. This result is consistent with the previous one (26). The results of correlation analysis showed that PC and PC/MPV had a good correlation with sFlt-1 and PlGF, suggesting that $\mathrm{PC}$ and $\mathrm{PC} / \mathrm{MPV}$ can be used as potential markers for assessing the risk of PE. Lecarpentier et al. (27) emphasized the important value of the sFlt-1/PlGF ratio in predicting adverse pregnancy outcomes, with an AUC of 0.76. In our study, the AUC of sFlt-1/PIGF was higher than either alone, suggesting that sFlt-1/PlGF has a higher predictive value for the occurrence of $\mathrm{PE}$ in women in the second trimester. It has been suggested that sFlt-1/PlGF is insufficient to predict the risk of PE, and other reliable indicators are needed, of which platelet parameters have the advantages of easy, quick, and low cost (28). De Moreuil et al. (29) analyzed the ROC curve for PC, MPV, PDW, and $\mathrm{PC} / \mathrm{MPV}$ and found that these parameters have predictive value. Abdel et al. (30) found that platelet parameters during the second trimester were higher in women who subsequently developed PE, but MPV and PDW had low predictive value and were not a good predictor of PE. In our study, ROC curve analysis showed that PC/MPV had a higher ability to predict $\mathrm{PE}$, which was consistent with the above study.

Moreover, AlSheeha et al. (31) reported that women who eventually developed PE had a significant decrease in PC/ MPV at 20-24 weeks of gestation, and a similar change in $\mathrm{PC} / \mathrm{MPV}$ was observed in this study. Compared with the control group, the PC/MPV of the PE group was lower, and the AUC was 0.836, indicating that platelet parameters were helpful to predict the occurrence of $\mathrm{PE}$ in time. However, the accuracy of PC/MPV is still not ideal and a combination of more indicators will be helpful for the prediction of PE. ROC curve analysis was conducted to further evaluate the utility of PC/MPV combined with sFlt-1, PlGF, and sFlt-1/PlGF. The results showed that the AUC of PC/MPV combined with sFlt-1/PlGF was the highest, which means that the combination of PC/MPV and sFlt-1/ PIGF has the best predictive ability for PE.

This study has some limitations. First, this study sample size was relatively small and it was a single-center study. In the future, the sample size needs to be increased 
to strengthen our results. Second, the pregnant women included in the study were at a specific gestational stage, and further studies evaluating women during the whole pregnancy are required.

\section{Conclusions}

In summary, $\mathrm{PC} / \mathrm{MPV}$ is a useful candidate for the prediction of $\mathrm{PE}$ and can significantly improve the prediction ability of sFlt-1 and PlGF. The use of sFlt-1/ PlGF combined with PC/MPV in pregnant women at 20 24 weeks of gestational age has important clinical value for the early prediction and treatment of PE.

\section{Acknowledgments}

The authors would like to thank the staff and participants for their contribution to this study.

Funding: The study was funded by the WU JIEPING Medical Foundation (320.6750. 18118).

\section{Footnote}

Reporting Checklist: The authors have completed the STARD reporting checklist. Available at https://dx.doi. org/10.21037/apm-21-1244

Data Sharing Statement: Available at https://dx.doi. org/10.21037/apm-21-1244

Conflicts of Interest: Both authors have completed the ICMJE uniform disclosure form (available at https://dx.doi. org/10.21037/apm-21-1244). The authors have no conflicts of interest to declare.

Ethical Statement: The authors are accountable for all aspects of the work in ensuring that questions related to the accuracy or integrity of any part of the work are appropriately investigated and resolved. All procedures performed in this study involving human participants were in accordance with the Declaration of Helsinki (as revised in 2013). The study was approved by the Ethics committee of Beijing Jishuitan Hospital and informed consent was taken from all the patients.

Open Access Statement: This is an Open Access article distributed in accordance with the Creative Commons Attribution-NonCommercial-NoDerivs 4.0 International
License (CC BY-NC-ND 4.0), which permits the noncommercial replication and distribution of the article with the strict proviso that no changes or edits are made and the original work is properly cited (including links to both the formal publication through the relevant DOI and the license). See: https://creativecommons.org/licenses/by-nc-nd/4.0/.

\section{References}

1. Ives CW, Sinkey R, Rajapreyar I, et al. PreeclampsiaPathophysiology and Clinical Presentations: JACC Stateof-the-Art Review. J Am Coll Cardiol 2020;76:1690-702.

2. Bokslag A, van Weissenbruch $M$, Mol BW, et al. Preeclampsia; short and long-term consequences for mother and neonate. Early Hum Dev 2016;102:47-50.

3. Dymara-Konopka W, Laskowska M, Oleszczuk J. Preeclampsia - Current Management and Future Approach. Curr Pharm Biotechnol 2018;19:786-96.

4. Le Y, Ye J, Lin J. Expectant management of early-onset severe preeclampsia: a principal component analysis. Ann Transl Med 2019;7:519.

5. Serra B, Mendoza M, Scazzocchio E, et al. A new model for screening for early-onset preeclampsia. Am J Obstet Gynecol 2020;222:608.e1-18.

6. Yoffe L, Gilam A, Yaron O, et al. Early Detection of Preeclampsia Using Circulating Small non-coding RNA. Sci Rep 2018;8:3401.

7. Zhang M, Wan P, Ng K, et al. Preeclampsia Among African American Pregnant Women: An Update on Prevalence, Complications, Etiology, and Biomarkers. Obstet Gynecol Surv 2020;75:111-20.

8. Correa PJ, Palmeiro Y, Soto MJ, et al. Etiopathogenesis, prediction, and prevention of preeclampsia. Hypertens Pregnancy 2016;35:280-94.

9. Dröge LA, Perschel FH, Stütz N, et al. Prediction of Preeclampsia-Related Adverse Outcomes With the sFlt1 (Soluble fms-Like Tyrosine Kinase 1)/PIGF (Placental Growth Factor)-Ratio in the Clinical Routine: A RealWorld Study. Hypertension 2021;77:461-71.

10. Neuman RI, van der Meer MMA, Saleh L, et al. Copeptin and mid-regional pro-atrial natriuretic peptide in women with suspected or confirmed pre-eclampsia: comparison with sFlt-1/PIGF ratio. Ultrasound Obstet Gynecol 2020;56:872-8.

11. Herraiz I, Simón E, Gómez-Arriaga PI, et al. Clinical implementation of the sFlt-1/PlGF ratio to identify preeclampsia and fetal growth restriction: A prospective cohort study. Pregnancy Hypertens 2018;13:279-85. 
12. Moradi MT, Rahimi Z, Vaisi-Raygani A. New insight into the role of long non-coding RNAs in the pathogenesis of preeclampsia. Hypertens Pregnancy 2019;38:41-51.

13. Stepan H, Hund M, Andraczek T. Combining Biomarkers to Predict Pregnancy Complications and Redefine Preeclampsia: The Angiogenic-Placental Syndrome. Hypertension 2020;75:918-26.

14. Nobakht MGBF. Application of metabolomics to preeclampsia diagnosis. Syst Biol Reprod Med 2018;64:324-39.

15. De Kat AC, Hirst J, Woodward M, et al. Prediction models for preeclampsia: A systematic review. Pregnancy Hypertens 2019;16:48-66.

16. Denhez B, Wang L, Moreau J, et al. Interlaboratory bias of albuminuria and proteinuria in hypertensive pregnancy. Clin Biochem 2021;87:13-8.

17. Bayoumi MAA, Ali AAH, Hamad SG, et al. Effect of Maternal Preeclampsia on Hematological Profile of Newborns in Qatar. Biomed Res Int 2020;2020:7953289.

18. Ekun OA, Ogidi NO, Lawal RA, et al. Interrelationship Between Markers of Oxidative Stress, Inflammation and Hematological Parameters Among Preeclamptic Nigerian Women. Med Sci Monit Basic Res 2018;24:225-31.

19. Jakobsen C, Larsen JB, Fuglsang J, et al. Platelet function in preeclampsia - a systematic review and meta-analysis. Platelets 2019;30:549-62.

20. Morton A. Imitators of preeclampsia: A review. Pregnancy Hypertens 2016;6:1-9.

21. Quan LM, Xu Q L, Zhang GQ, et al. An analysis of the risk factors of preeclampsia and prediction based on combined biochemical indexes. Kaohsiung J Med Sci 2018;34:109-12.

22. Bramham K, Parnell B, Nelson-Piercy C, et al. Chronic hypertension and pregnancy outcomes: systematic review and meta-analysis. Bmj 2014;348:g2301.

23. Zeisler H, Llurba E, Chantraine F, et al. Predictive Value of the sFlt-1:PlGF Ratio in Women with Suspected Preeclampsia. N Engl J Med 2016;374:13-22.

24. Eastabrook G, Aksoy T, Bedell S, et al. Preeclampsia biomarkers: An assessment of maternal cardiometabolic health. Pregnancy Hypertens 2018;13:204-13.

25. Perales A, Delgado JL, de la Calle M, et al. sFlt-1/PlGF for prediction of early-onset pre-eclampsia: STEPS (Study of Early Pre-eclampsia in Spain). Ultrasound Obstet Gynecol 2017;50:373-82.

26. Cui L, Shu C, Liu Z, et al. The expression of serum sEGFR, sFlt-1, sEndoglin and PLGF in preeclampsia. Pregnancy Hypertens. 2018;13:127-132

27. Lecarpentier E, Tsatsaris V. Angiogenic balance (sFlt1/PlGF) and preeclampsia. Ann Endocrinol (Paris). 2016;77:97-100.

28. Liu Y, Zhao Y, Yu A, et al. Diagnostic accuracy of the soluble Fms-like tyrosine kinase-1/placental growth factor ratio for preeclampsia: a meta-analysis based on 20 studies. Arch Gynecol Obstet 2015;292:507-18.

29. de Moreuil C, Herry E, Lacut K, et al. Correlation of biological parameters with placental parameters and pregnancy outcomes in pre-eclamptic women. Pregnancy Hypertens 2020;19:61-6.

30. Abdel Razik M, Mostafa A, Taha S, et al. Combined Doppler ultrasound and platelet indices for prediction of preeclampsia in high-risk pregnancies. J Matern Fetal Neonatal Med 2019;32:4128-32.

31. AlSheeha MA, Alaboudi RS, Alghasham MA, et al. Platelet count and platelet indices in women with preeclampsia. Vasc Health Risk Manag 2016;12:477-80.

(English Language Editor: B. Draper)
Cite this article as: Li Y, Sun L, Zheng X, Liu J, Zheng R, Lv Y. The clinical value of platelet parameters combined with sFlt-1/PlGF in predicting preeclampsia. Ann Palliat Med 2021;10(7):7619-7626. doi: 10.21037/apm-21-1244 